\title{
An Evaluation of Physician Predictions of Discharge on a General Medicine Service
}

\author{
Brian Sullivan, MD¹, David Ming, MD²,3, Joel C. Boggan, MD, MPH ${ }^{1,4}$, Ryan D. Schulteis, MD ${ }^{1,4}$, Samantha Thomas, MB ${ }^{5}$ \\ Josh Choi, MD1, Jonathan Bae, MD2*
}

\begin{abstract}
${ }^{1}$ Department of Medicine, Duke University Medical Center, Durham, North Carolina; ${ }^{2 H o s p i t a l ~ M e d i c i n e, ~ D e p a r t m e n t ~ o f ~ M e d i c i n e, ~ D u k e ~ U n i v e r s i t y ~}$ Medical Center, Durham, North Carolina; ${ }^{3}$ Division of Hospital \& Emergency Medicine, Department of Pediatrics, Duke University Medical Center, Durham, North Carolina; ${ }^{4}$ Department of Medicine, Durham Veterans Affairs Medical Center, Durham, North Carolina; ${ }^{5}$ Department of Biostatistics and Bioinformatics, Duke University, Durham, North Carolina.
\end{abstract}

The goal of this study was to evaluate general medicine physicians' ability to predict hospital discharge. We prospectively asked study subjects to predict whether each patient under their care would be discharged on the next day, on the same day, or neither. Discharge predictions were recorded at 3 time points: mornings (7-9 AM), midday (12-2 PM), or afternoons (5-7 PM), for a total of 2641 predictions. For predictions of next-day discharge, the sensitivity (SN) and positive predictive value (PPV) were lowest in the morning ( $27 \%$ and $33 \%$, respectively), but increased by the afternoon (SN 67\%, PPV 69\%). Similarly, for same-day discharge predictions, SN and PPV were highest at midday ( $88 \%$ and $79 \%$, respectively). We found that although physicians have difficulty predicting next-day discharges in the morning prior to the day of expected discharge, their ability to correctly predict discharges continually improved as the time to actual discharge decreased. Journal of Hospital Medicine 2015;10:808-810. (c) 2015 Society of Hospital Medicine
Hospital discharge planning is a complex process requiring efficient coordination of many different medical and social support services. For this reason, multidisciplinary teams work together to develop individualized discharge plans in an attempt to reduce preventable adverse events related to hospital discharge. ${ }^{1-5}$ Despite these ongoing efforts, optimal discharge strategies have yet to be realized. ${ }^{1,4-9}$

One factor that may improve the discharge process is the early identification of patients who are approaching discharge. ${ }^{10}$ Multidisciplinary teams cannot fully deploy comprehensive discharge plans until a physician deems a patient to be approaching discharge readiness. 8

To our knowledge, no studies have examined the performance of physician predictions of upcoming discharge. Instead, prior studies have found that physicians have difficulty predicting the length of stay for patients seen in the emergency room and for elderly patients newly admitted to general medicine floor. ${ }^{11,12}$ The purpose of this study was to evaluate the ability of inpatient general medicine physicians to predict next or same-day hospital discharges to help inform the timing of discharge planning.

*Address for correspondence and reprint requests: Jonathan Bae, MD, Duke University Medical Center, Box 100800, Durham, NC 27710; Telephone: 919-681-8263; Fax: 919-668-5394; E-mail: jon.bae@dm. duke.edu

Additional Supporting Information may be found in the online version of this article.

Received: March 15, 2015; Revised: June 10, 2015; Accepted: June 17,2015

2015 Society of Hospital Medicine DOI 10.1002/jhm.2439

Published online in Wiley Online Library (Wileyonlinelibrary.com).

\section{METHODS}

We collected daily in-person predictions from all senior residents and attendings separately on the inpatient general medicine teams $(5$ resident/attending services and 4 attending-only services) at a single 950-bed academic medical center. We asked these physicians to predict whether each patient under their care had a greater than or equal to $80 \%$ chance of being discharged the next day, the same day, or neither (ie, no discharge on the next or same day).

Physician predictions of discharge occurred Monday through Friday at 1 of 3 time points: morning (7-9 AM), midday (12-2 PM), or afternoon (5-7 PM). Data collection focused on 1 time point per week during 2 different weeks in November 2013 and 1 week in February 2014. Predictions of same-day discharge could only be made at the morning and midday time points. Each patient could have multiple predictions if they remained hospitalized during subsequent assessments. For each physician making a prediction, we recorded the physician training level (resident or attending).

This protocol was deemed exempt by our university's institutional review board.

\section{Outcomes}

We measured the sensitivity (SN), specificity (SP), positive predictive value (PPV), and negative predictive value (NPV) for each type of physician prediction (next day, same day, or no discharge by the end of the next day). We also calculated these measurements for each time point in the time of day subgroup: morning, midday, and afternoon. 


\section{Statistical Analyses}

Using a normal approximation to the binomial distribution, point estimates and $95 \%$ confidence intervals for SN, SP, PPV, and NPV for the group of all patients and for the time of day subgroup are reported. The Cochran-Armitage trend test was used to examine trends in SN, SP, PPV, and NPV as time to discharge decreased. No adjustments were made for multiple comparisons. A 2-sided significance level was prespecified at 0.05 for all tests.

For the subset of patients who had discharge predictions made by both a resident and an attending, agreement was examined using the kappa statistic. All analyses were conducted using SAS version 9.3 (SAS Institute, Cary, NC).

\section{RESULTS}

A total of 2660 predictions were made by 24 attendings and 15 residents. Nineteen predictions were excluded because of missing prediction type or date of discharge, leaving 2641 predictions for analysis. Table 1 summarizes the total number of predictions within subgroups.

The overall daily discharge rate in our population was $22.3 \%$ (see Supporting Table 1 in the online version of this article for the raw values). The SN and PPV of physician predictions of next-day discharge were $48 \%$ (95\% confidence interval [CI]: $43 \%-52 \%$ ) and $51 \%$ (95\% CI: 46\%-56\%), respectively. The SN and PPV for same-day discharge predictions were

\begin{tabular}{lc}
\hline TABLE 1. Summary of Predictions & \\
\hline & No. of Predictions \\
\hline All predictions & 2,641 \\
Day of the week & \\
Monday & 596 \\
Tuesday & 503 \\
Wednesday & 525 \\
Thursday & 551 \\
Friday & 466 \\
Physician training level & \\
Resident & 871 \\
Attending & 1,770 \\
Time of day & \\
Morning (7 AM-9 AM) & 906 \\
Midday (12 PM-2 PM) & 832 \\
Afternoon (5 PM-7 PM) & 903
\end{tabular}

73\% (95\% CI: 68\%-78\%) and 69\% (95\% CI: 64\%$73 \%)$, respectively. The SP for next and same-day discharge predictions was 90\% (95\% CI: 89\%-91\%) and $95 \%$ (95\% CI: $94 \%-96 \%$ ), whereas the NPV was $89 \%$ (95\% CI: $88 \%-90 \%$ ) and $96 \%$ (95\% CI: $95 \%-$ $97 \%)$, respectively.

Outcome measures for each prediction type are stratified by time of day and summarized in Table 2 . For next-day discharge predictions, the SN and PPV were lowest in the morning (SN 27\%, PPV 33\%) and peaked by the afternoon (SN 67\%, PPV 69\%). Similarly, for same-day discharges, SN and PPV were highest later in the day (midday SN 88\%, PPV 79\%). This trend is also demonstrated in the SP and NPV, which increased as time to actual discharge approached, although the trends are not as pronounced as for $\mathrm{SN}$ and PPV.

The overall agreement between resident and attending predictions was measured and found to have kappa values of $0.51(P<0.001)$ for next-day predictions and $0.73(P<0.001)$ for same-day predictions, indicating moderate and substantial agreement, respectively (see Supporting Table 2 in the online version of this article). ${ }^{13}$

\section{DISCUSSION}

This is the first study, to our knowledge, to examine the ability of physicians to predict upcoming discharge during the course of routine general medicine inpatient care. We found that although physicians are poor predictors of discharge in the morning prior to the day of expected discharge, their ability to correctly predict inpatient discharges showed continual improvement as the difference between the prediction time and time of actual discharge shortened.

For next-day predictions, the most accurate time point was the afternoon, when physicians correctly predicted more than two-thirds of actual next-day discharges. This finding suggests that physicians can provide meaningful discharge estimates as early as the afternoon prior to expected discharge. This may be an optimal time for physicians to meet with the multidisciplinary discharge teams, as many preparations hinge on timely and accurate predictions of discharge (eg, arranging patient transportation, postdischarge visits by a home health company). Multidisciplinary teams will also be reassured that an afternoon prediction of

TABLE 2. Results by Discharge Prediction Type and Time of Day

\begin{tabular}{|c|c|c|c|c|c|c|c|c|}
\hline \multirow[b]{2}{*}{ Validity Measure } & \multicolumn{3}{|c|}{ Next-Day Discharge Predictions } & \multirow[b]{2}{*}{ Trend $P$ Value } & \multicolumn{3}{|c|}{ Same-Day Discharge Predictions } & \multirow[b]{2}{*}{ Trend $P$ Value } \\
\hline & Morning & Midday & Afternoon & & Morning & Midday & Afternoon & \\
\hline Sensitivity & $0.27(0.21-0.35)$ & $0.50(0.41-0.59)$ & $0.67(0.59-0.74)$ & $<0.001$ & $0.66(0.59-0.73)$ & $0.88(0.81-0.93)$ & - & $<0.001$ \\
\hline Specificity & $0.87(0.85-0.90)$ & $0.90(0.88-0.92)$ & $0.93(0.91-0.95)$ & $<0.001$ & $0.88(0.85-0.90)$ & $0.95(0.93-0.97)$ & - & $<0.001$ \\
\hline PPV & $0.33(0.25-0.41)$ & $0.48(0.40-0.57)$ & $0.69(0.61-0.76)$ & $<0.001$ & $0.62(0.55-0.68)$ & $0.79(0.71-0.85)$ & - & $<0.001$ \\
\hline NPV & $0.84(0.81-0.87)$ & $0.91(0.88-0.93)$ & $0.93(0.91-0.94)$ & $<0.001$ & $0.90(0.88-0.92)$ & $0.98(0.96-0.99)$ & - & $<0.001$ \\
\hline
\end{tabular}


next-day discharge would only prematurely activate discharge resources in roughly 3 out of every 10 occurrences. Even in these instances, patients may benefit from the extra time for disease counselling, medication teaching, and arrangement of home services. $^{4-9}$

This investigation has several limitations. Our study was conducted at a large tertiary care center over brief time periods, with an overall discharge rate of about 1 in 5 patients per day. Thus, the results may not be generalizable to hospitals with different patient populations, volume, or turnover, or when predictions are made at different times throughout the year. Furthermore, we were unable to determine if the outcome measures were affected by prolonged lengths of stay or excessive predictions on relatively few patients. However, we sought to mitigate these constraints by surveying many different respondents with varying experience levels, caring for a heterogeneous patient population at nonconsecutive time points during the year. A review of our hospital's administrative data suggests that the bed occupancy and average length of stay during our surveys were similar with most other time points during the year, and therefore representative of a typical inpatient general medicine service.

Our investigation was a novel investigation into the performance of physician discharge predictions, which are daily predictions made either explicitly or implicitly by physicians caring for patients on a general medicine ward. By utilizing a simple, subjective survey without bulky calculations, this approach closely mirrors real-world practice patterns, and if further validated, could be easily assimilated into the normal workflow of a wide range of busy clinicians to more effectively activate individualized discharge plans. ${ }^{1-5}$

Future work could capture additional patient information, such as functional status, diagnosis, and current length of stay, which would allow identification of certain subsets of patients in which physicians are more or less accurate in predicting hospital discharge. Additionally, the outcomes of "incorrect" predictions, particularly the "surprise discharges" that left even though they were predicted to stay, could be assessed.
If patients were discharged prematurely, this may be reflected by a higher 30-day readmission rate, lower clinic follow-up rate, and/or lower patient satisfaction scores.

\section{CONCLUSION}

Although physicians are poor predictors of discharge in the morning prior to the day of expected discharge, their ability to correctly predict inpatient discharges steadily improves as the difference between the prediction time and time of actual discharge shortened. It remains to be determined if systematic incorporation of physician discharge predictions into standard workflows will improve the effectiveness of transition of care interventions.

Disclosure: Nothing to report.

\section{References}

1. Brock, J, Mitchell, J, Irby, K, et al. Care transitions project team: Association between quality improvement for care transition in communities and rehospitalizations among Medicare beneficiaries. JAMA. 2013;309(4):381-391.

2. Coleman EA, Parry C, Chalmers S, Min SJ, The care transitions intervention: results of a randomized controlled trial. Arch Intern Med. 2006;166:1822-1828.

3. Naylor MD, Brooten D, Campbell R, et al. Comprehensive discharge planning and home follow-up of hospitalized elders: a randomized clinical trial. JAMA. 1999;281:613-620.

4. Rennke S, Nguyen OK, Shoeb MH, et al. Hospital-initiated transitional care interventions as a patient safety strategy: a systematic review. Ann Intern Med. 2013;158:433-440.

5. Shepperd S, McClaren J, Phillips CO, et al. Discharge planning from hospital to home. Cochrane Database Syst Rev. 2013;1:CD000313.

6. Carey MR, Sheth H, Braithwaite SR. A prospective study of reasons for prolonged hospitalizations on a general medicine teaching service. J General Intern Med. 2005;20:108-115.

7. Selker HP, Beshansky JR, Pauker SG, Kassirer JP. The epidemiology of delays in a teaching hospital. Med Care. 1989;27:112-129.

8. Soong C, Daub S, Lee J, et al. Development of a checklist of safe discharge practices for hospital patients. J Hosp Med. 2013;8:444-449.

9. Kwan JL, Lo L, Sampson M, Shojania KG, Medication reconciliation during transition of care as a patient safety strategy. Ann Intern Med. 2013;158:397-403.

10. Webber-Maybank M, Luton H, Making effective use of predicted discharge dates to reduce the length of stay in hospital. Nurs Times. $2009 ; 105(15): 12-13$

11. Asberg KH. Physicians' outcome predictions for elderly patients: Survival, hospital discharge, and length of stay in a department of internal medicine. Scand J Soc Med. 1986;14(3):127-132.

12. Mak G, Grant WD, McKenzie JC, McCabe JB. Physicians' ability to predict hospital length of stay for patients admitted to the hospital from the emergency department. Emerg Med Int. 2012;2012:824674.

13. Viera AJ, Garrett JM. Understanding interobserver agreement: the kappa statistic. Fam Med. 2005;37(5):360-363. 